\title{
Impacto de las afecciones genéticas en lactantes desnutridos secundarios
}

\author{
Fanny Cortés M. ${ }^{1} ;$ M. Angélica Alliende R.2
}

\section{Genetic and congenital disorders among infants with secondary undernutrition}

\begin{abstract}
Children with secondary undernutrition are being detected with increased frequency in Chile in the last 20 ycors, duc ic effactive control measures tor primary malnurilion. Among 1542 patients under two years of age who were admilled :o a center for the recovery and study of children with sccondary undernutrition, from january 1935 throughoul cecember 1990, adsctiption of each one of them to one of the diagnostic cothegories of Hollp from the start point of clinical d agriss is at admission, showed thal genelic disorders and congenital malformations were the main couse $\mid 40.1 \% ;$ al inc admissions idefinitely genetic diseases 12.9\%; poligenicultifaclorial disorders $15.8 \%$; developmental anomalies $9.1 \%$ diseases due to leralcgens $2.3 \%$. Fitty four cases had chromosomal abnomalilies, sugesling the need of rylogenclik: sludes in oatients with secondary undernutrilion plus dysmorphogenic defects or developmental retardation.
\end{abstract}

¡Key words: abnomalilies, birth defecis, congenital defects, chromosome, gen, diug induced, secondaiy undernutrilion.!

El crecimiento y desarrollo de un niño están determinados por la interacción de factores genéticos y ambientales, por lo tanto, una vez que los requerimientos están satisfechos en forma proporcionada y continua, una constitución genética adecuada es condición necesaria para el crecimiento y desarrollo nomal ${ }^{1}$. En los países subdesarrollados, sectores importantes de la población se ven afectados por múltiples e intensas restricciones ambicntales. Esta situación de privación constituye la principal determinante de la patología prevalente y de las diferencias observadas en crecimiento y desarrollo?

Durante los áltimos 20 años, mediante la aplicación de programas específicos de alimentación complementaria y a la creación de centros cerrados de recuperación nutricional, se ha logtado una disminución progresiva de las tasas de desnutrición infantil ${ }^{3-6}$. Los mencionados programas han permitido el control de la desnutrición primaria y la detección de afecciones no nutri-

1. Médico. Unidad de Genética y Enfemedades Metabó licas, INTA, Universidad de Chile.

2. M.Sc. Lnidad de Genética y Enfemedades Metabólicas, INT A. Universidad de Chile. cionales que producen, secundariamente, delerioro del crecimiento. El aumento progresivo de lactantes desnutridos secundarios en los centros de la Corporación Nacional para la Nutrición Infantil (CONIN), motivó la creación de centros de recuperación y estudio de desnutridos secundarios (CREDES), cuyo objetivo principal es identificar la causa del deterioro de la nutrición, controlarla si es posible, logrando la recuperación nutricional del paciente.

Estudios previos, efectuados en pocos casos $y$ antes de la creación de CREDES, mostraron que el númcro de individuos que presentaba una desnutrición de causa genética era importante ${ }^{1}$. El presente trabajo pretende corroborar los datos anteriores en el sentido de determinar, con un mayor número de casos, el impacto de las afecciones genéticas en la desnulrición secundaria, clasificar las afecciones genćticas en diferentes categorías y determinar el impacto relativo de cada una.

\section{Material y Método}

Se revisaron las fichas clínicas de todos los pacientcs ingresados a CREDES entre encro de 1985 y diciembre de 1990. Según el diagnostico formulado en la admisión, cada 
paciente fue incurporado a una de las categorías de diagnosrico descritas por Hall?, que clasifica las enfermedades, según su origen, en: clararnente genéticas (cuando su etiología está bien establecida, ya sea monogénica o cromosómica), poligénicas-multifacivriales (las que estudjadas en profundidad han mostrado estar determinadas por múltiples genes y sus adcios), anomalías del desarrolio (afecciones consideradas malfomaciones producidas por anomalías en cl desarollo embrionario de etiologla desconocila y para las cuales, hasta la fecha, no se ha establecido un diagnóstico de recurrencia específico), teratógenos (anomalías del desartollo cmbnonario producidas por la acción de un agentc conocido) y anomalías no genćticas (cuando la etiología carcce de tal base). Se realizaron 124 estudios cromosómicos, en pacicntes que además de su desnutrición presentaban retraso del desarrollo psicomotor, múltiples malformaciones congénitas o dismorfias no características de una afección monogénica especifica. Los cromosomas fueron sometidos a bandeo GTG (Clici cuando había indicación). En los 70 primeros se utilizaron las técnicas corrientes descritas para estudio de linfocitos; en los 54 últimos se agregó bromuso de etilo en las dos últimas horas de cultivo para lograr mejor resolución dc handas $5^{3}$. Fn las alecciones monogénicas, los diagnósticos fucron asignados scgún la catcgoría respectiva del calálogo de afecciones mendelianas de McKusick'0.

\section{Resultados}

Durante el periodo mencionado se analizaron 1542 pacientes desnutridos, entre los cuales las afccciones claramente genéticas correspondian a $12,9 \%$ (n: 199), las poligenicas-multifactoriales 15,8\% (n: 243), las anomalías del desartol lo 9,1\% (n: 14 I) y los teralógenos $2,3 \%(\mathrm{n}: 35)$ (figura l), de los diagnósticos de egreso, representando en conjunto $40,1 \%$ de los casos.

Entre las afecciones claramente genćticas, $26 \%$ (n: 52) eran abcrraciones cromosómicas; $42,5 \%$ (n: 84) enfermedades hereditarias autosómicas recesivas; $25 \%$ (n: S0) autosómicas dominantes y $6,5 \%$ (n: 13) ligadas al cromosoma $X$ (ligura 2).

La tabla 1 muestra las aberraciones cromosómicas encontradas. De éstas, $75 \%$ eran alteraciones numéricas, principalmente trisomía 21 , corespondiendo el $25 \%$ restante a alteraciones estructurales poco frecuentes en la población general.

En1re los diagnósticos específicos establecidos con mayor periodicidad destaca la alta frecuencia de malformaciones cardiovasculares y del tipo de herencia poligénico-multifactorial, como se muestra en la tabla 2.

\section{Comentario}

La frecuencia de afecciones genéticas encontrada, es menor que la descrita en pacientes pediátricos hospitalizados, cercana a $60 \%$ cn países desarrollados 1, 2.:1-14. Esto se debe a que en esta serie no se incluyeron las que no producen deterioro de la nutrición y que estaban presentes ел varios pacientes del estudio (por cjemplo enfermedad de Von Willebrand, esferocitosis, displasia de caderas, escoliosis), cuya inclusión podría accrca las proporciones a las señaladas.

El hallazgo de un número considerable de aberraciones cromosomicas poco frecuentes en la población general, demucstra la necesidad de hacer estudios citogenécicos a todos los pacicntes con desnutrición secundaria asociada a dismorlias o retraso del desarrollo psicomotor. Es así como

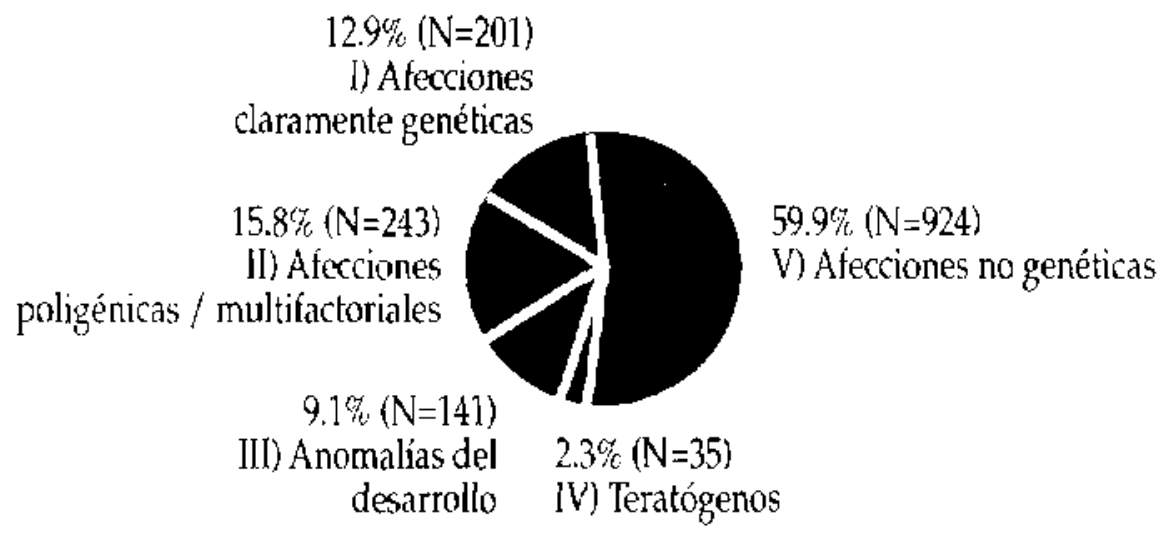

Figura 1: Frecucncia de afecciones genéticas y no genéticas. 


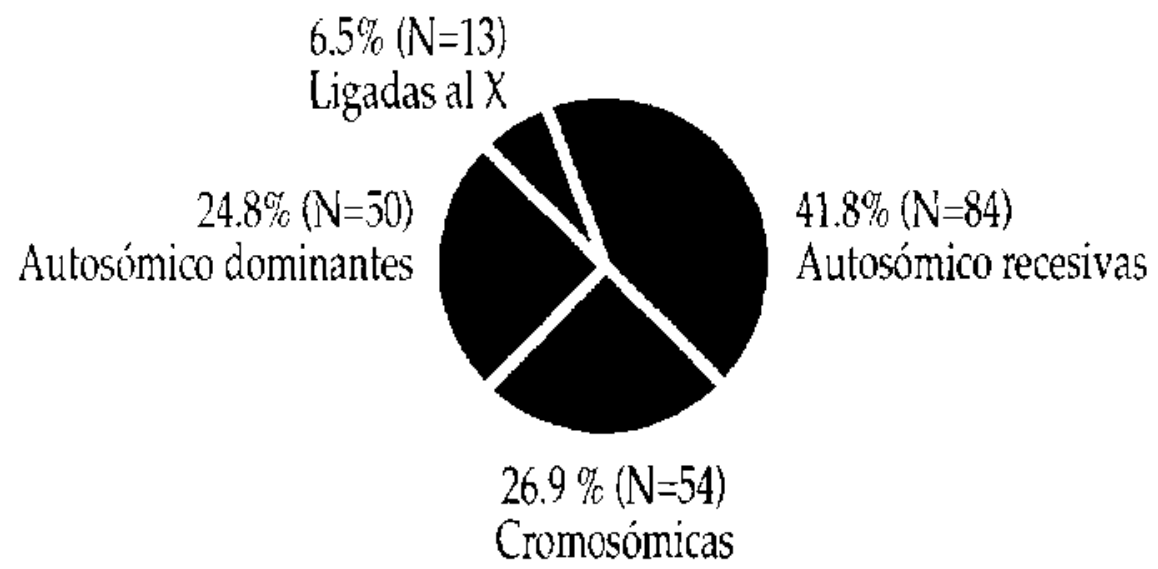

Figura 2: Frecuencia de las afeccioncs claramente genćlicas.

Tabla 1

\begin{tabular}{|c|c|}
\hline Alteraciones & \\
\hline Diagnóstico & $\mathbf{n}$ \\
\hline \multicolumn{2}{|l|}{ Alterackones numéricas } \\
\hline $\begin{array}{l}47, X X \circ X Y,+21 \\
45, X \\
47, X Y+18 \\
47, X X+13 \\
47, X X+22 q\end{array}$ & $\begin{array}{r}39 \\
3 \\
1 \\
1 \\
1\end{array}$ \\
\hline Sublotal & 45 \\
\hline \multicolumn{2}{|l|}{ Alteraciones estructurales } \\
\hline $\begin{array}{l}\text { 46, XX o XY del (5p) } \\
46, X X, \text { del }(4 p) \\
46, X X, \text { inv }(6) \\
46, X Y, \text { del }(18 p) \\
46, X X, r(18) \\
46, X X, \text { del }(22 p)\end{array}$ & $\begin{array}{l}3 \\
2 \\
1 \\
1 \\
1 \\
1\end{array}$ \\
\hline Subtolal & 9 \\
\hline Total & 54 \\
\hline
\end{tabular}

$s \mathrm{c}$ ha fogrado establecer cl diagnóstico de certcza en la gran mayoría de los pacientes $(89,5 \%)$, sin embargo, hay aún en la categoría de anomalias del desartollo un número importante de individuos con múltiples anomalías congénitas $(10,5 \%)$, en los que no se ha llegado a uno específico. En la medida que su estudio se vaya completando, es posible que estos individuos puedan ser asignados a categorías más espccíficas de afecciones cromosómicas o monogénicas.

Finalmente, si bien las perspectivas terapéliticas de las enfermedades genéticas son muy promisorias gracias a los avances de la genética molecular, la práctica actual de la genética médica es básicamente preventiva y esta prevención. preconcepcional, apoyada en el consejo genćtico, requicre un diagnóstico de ccrteza y hacia allá se deben dirigir nucstros esfuerzos.

\section{Resumen}

El control de las tasas de desnutrición primaria en Chile ha permitido la detección de un número creciente de lactantes con desnutrición secundaria, como lo confirma esta investigación realizada entre los 1542 lactantes ingresados a un centro de recuperación y esludio de desnutridos securidarios, en el período comprendido entre enero de 1985 y diciembre de 1990 . Cada uno de los pacientes fuc asignado, de acuerdo con $\mathrm{cl}$ diagnóstico de ingreso, a una de las categorias de la clasificación de Hall, según la cual las afecciones genéticas y las malformaciones congénitas eran responsables de $40,1 \%$ de los ingrcsos (afecciones ciertamente genéricas $12,9 \%$, enfermedades poligénicas-multifactoriales $15,8 \%$, anomalías del desarrollo $9,1 \%$, causadas por terâtúgenos $2,3 \%$ ). EJ hallazgo de un número considerable de anomalías cromosómicas (n: 54), en mayor proporción que en la población gene- 
Tabla 2

Diagnósticos mús frecuentes y tipo de herencia

\begin{tabular}{|c|c|c|}
\hline Diagnóstlco & $\mathbf{n}$ & $\begin{array}{l}\text { Tipo de } \\
\text { herencia }\end{array}$ \\
\hline Cardiopatía congénita & $\begin{array}{r}168 \\
30\end{array}$ & $\mathrm{PG} / \mathrm{MF}$ \\
\hline $\begin{array}{l}\text { Trisomía } 21 \\
\text { Sindrome de alcoholismo tetal }\end{array}$ & 30 & $\begin{array}{r}\mathrm{T} \\
\mathrm{T}\end{array}$ \\
\hline Fisura labio palatina & 21 & PG/MF \\
\hline En[ermedad de Hirschprung & 19 & $\mathrm{PG} / \mathrm{MF}$ \\
\hline Malformaciones del tracto urinario & 16 & $\mathrm{D}$ \\
\hline Microcefalia & 14 & D \\
\hline Enfermedad celíaca & 11 & $\mathrm{PG} / \mathrm{MF}$ \\
\hline Mielomeningocele & 10 & $\mathrm{PG} / \mathrm{MF}$ \\
\hline Secuencia de Picre Robin & 9 & D \\
\hline S. Silver Russell & 8 & AR \\
\hline Int. Cong. sacarosa-isomaltosa & 7 & AR \\
\hline
\end{tabular}

PG/MF: poligénico-multifactotial; CR: cromosómico: T: teratógeno; D: anomalía del desarrollo; AR: autosómicu recesivo.

ral, sugiere la conveniencia de realizar estudios citogenéticos ел los pacientes con desnutrición sccundaria y dismorfias o retardo psicomotor.

(Palabras clave: desnutrición secundaria, anomalias, defectos de nacimiento, defectos congénitos, cromosomas, genes, inducidos por đrogas, teratógenos.)

\section{Referencias}

1. Lacossie Y, Colombo M, López I: Desnutrición secundaria: impactu de las afecciones genéticas, metabólicas y neurologicas. Rev Chil Pediatr 1980; 51 : 257-260.
2. Monckeberg $F$ : Jaque al subdesarrollo. $3^{\text {Ed. Fititora }}$ Nacional Gabriela Mistral Ltda. Santiago, 1976.

3. Gonzólez N, Infante A: Programa de alimentación complententaria del sectur salud en Chile. Bol Sant Hanam 1980; 89: 563-571.

4. Atalah $E$, Puentes $R$, Castillo $D C$, Radrigán $M E$ : Prugrama nacional de alimenlación complementaria 1975. 1985. Kev Chil Yediatr 1985; 56: 362-368.

5. Monckeberg $F$ and Ruimalló $J$ : Nutrition recuvery centers. The chilean expericnce. En: Underwexd $\mathrm{B}$. Notrition intervention strategics in national development. Academic Press, 1983.

6. Informe del Gobiemo de Chile a la XXII Conferencia Satitatia Panamericana. 1986. Ministerio de Salud Santiago, Chile. Pp 63-68.

7. Hall JG, Powers EK, Mcllraine RT, Ean VII: 'Ihe frequency and financial burden of genetic discascs in a pediatric haspital. Am J Mcd Gener 1978; 1: 417 436.

8. Dutrillaux $B$, Confurier $J$ : La pratique de l'analyse chromosomique. Techniques de laboratoiries, Paris, Masson: 198I, 12:9.

9. Theuchi $T$ : Inhibitory effect of ethidjum bromide on milotic chromosome condensation and its application to high - resolution chromosome banding. Cytogenet Ccll Genet 1984; 38: 56-61.

10. McKusickVA: Mendelian inherilance in man. Cutalogs of autosomal dominant, autosomal rccessive and $\mathrm{X}$. linked phenotypes. 9th. Ed. Baitimore. John Hopkins Press, 1990.

11. Martínez-Frias ML, Frias $J L$, Saivador J. ClinicalEpidemiological Analysis of Malfomations. Am J Med Gene1 1989; 35: 121-125.

12. Coco $R_{1}$ Penchaszedeh VB: Cytogenetic findings in 200 children with mental retard ation and multiple congenital anornalies of unknowt cause. Am J Med Genet 1982; $12 \div 155-173$.

13. Imaizumi $Y H$, Yamamura $H$, Nishikawa $M_{2}$ Matuoka $M$ : The prevalence of birth of congenital malfomations at a matemily hospital in Osaka City. 1948-1990. Am J Hum Genet 1991; Suppl 49: 472.

14. Fitz Patrick OR, Skeoch Ch, Tolmie JL: Genetic aspects of admissions to a paediatric intensive care unit. Arch Dis Child 1991; 66:639-641. 